\title{
Remote-controlled exchange rates by photoswitchable internal catalysis of dynamic covalent bonds
}

\author{
David N. Barsoum, Julia A. Kalow* \\ Department of Chemistry, Northwestern University, Evanston, Illinois 60208, United States

\section{KEYWORDS}

\begin{abstract}
The transesterification of boronate esters with diols is tunable over at least 14 orders of magnitude. Rate acceleration is achieved by internal base catalysis, which lowers the barrier for the proton transfer step. Here we report a photoswitchable internal catalyst that tunes the rate of boronic ester/diol exchange over at least 4 orders of magnitude. We employed an acylhydrazone molecular photoswitch, which forms a thermally stable but photoreversible intramolecular $\mathrm{H}$-bond, to gate the activity of the internal base catalyst in 8-quinoline boronic esters. The photoswitch can be cycled repeatedly, with high photostationary states. The intramolecular $\mathrm{H}$ bond is found to be essential to the design of this photoswitchable internal catalyst, as protonating the quinoline with external sources of acid has little effect on the exchange rate.
\end{abstract}

Dynamic covalent chemistry (DCC) combines the strength and directionality of covalent bonds with the reversibility of supramolecular interactions. Owing to their tunability and robustness, dynamic covalent bonds have found wide application in library synthesis, bioconjugation, self-assembled receptors, covalent organic frameworks (COFs), self-healing and adaptive polymers, and responsive sensors. ${ }^{1-7}$ The formation and stability of these bonds are typically regulated by parameters such as temperature, $\mathrm{pH}$, concentration, catalyst loading, and light. Light is an ideal stimulus because it can be applied non-invasively with excellent spatial and temporal control. To design dynamic covalent bonds that respond to light, there must be a photoresponsive component. Photoswitches, which can be reversibly switched between two states using different wavelengths of light, offer a unique opportunity to remotely control DCC. ${ }^{8}$

Previously, photoswitches have been employed to govern the reactivity of dynamic covalent bonds via two principal strategies: (i) by rendering the dynamic bond active or inactive through lightdriven valence bond tautomerization; ${ }^{10-12}$ and (ii) by tuning the reactivity of the dynamic bond with an adjacent photoswitch. ${ }^{13,14}$ Notably, Hecht used azobenzene and spiropyran photoswitches to mask/unmask an activating hydroxyl group ortho to an aldehyde, which tunes the kinetics of imine formation with 2.4- and 3.1-fold differences in rates, respectively. ${ }^{14}$ Our group recently showed that azobenzene photoswitches can control the equilibrium of the boronic acid-diol condensation, due in part to the formation of intramolecular H-bonds. ${ }^{9}$ Here, we present a strategy to remotely control the kinetics of dynamic covalent reactions without affecting their thermodynamics, by designing a photoswitch that modulates

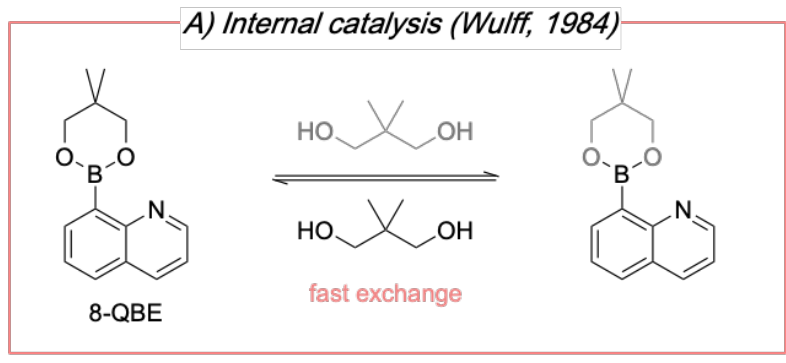

B) Photoswitchable H-bond (Hecht, 2015)

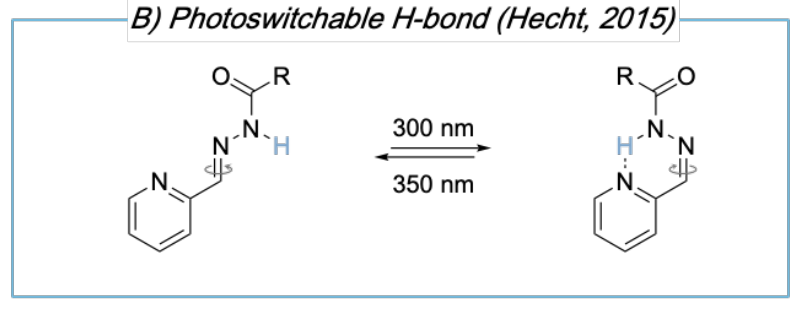

C) Photoswitchable internal catalysis (this work)

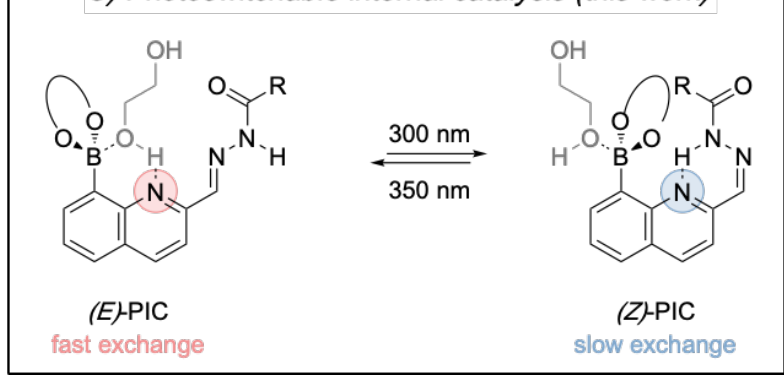

Figure 1. Conceptual design of photoswitchable internal catalysis, based on combining (A) the significant rate acceleration for boronic ester exchange provided by internal base catalysis and (B) bistable acylhydrazone photoswitches, yielding $(\mathrm{C})$ photoswitch-gated internal catalysis of the boronic ester exchange.

the reactivity of an internal catalyst. We have termed this approach photoswitchable internal catalysis (PIC). It should be noted that this is distinct from photoswitchable "external" catalysis, in which exogenous photoswitchable catalysts are introduced to modulate a structurally separate exchange reaction. ${ }^{15,16}$ Internal catalysis, in contrast, exploits proximity effects (neighboring-group participation) to dramatically alter the kinetics of a dynamic 
A)

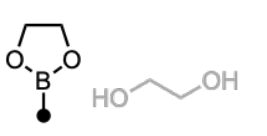

addition
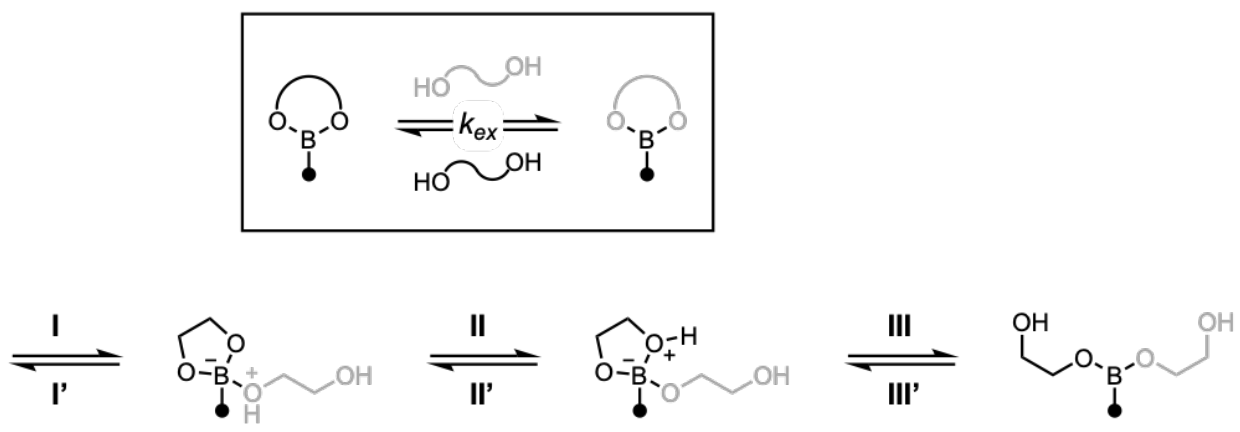

proton transfer

elimination

B)
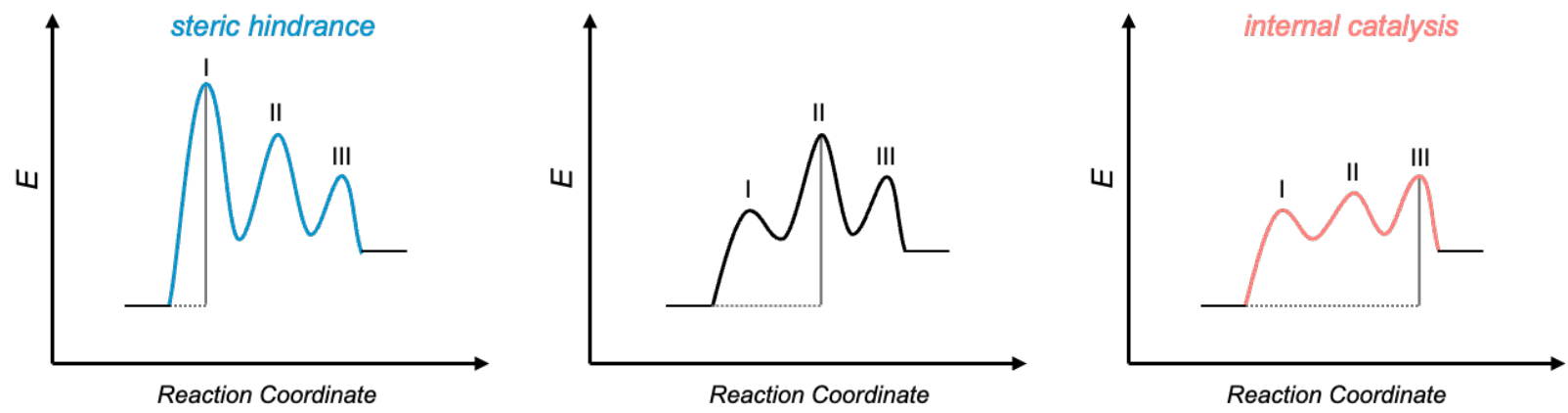

Figure 2. (A) Mechanism of degenerate exchange of boronate ester and free diol. The forward reaction (I $\rightarrow$ II $\rightarrow$ III) is followed by the reverse (III' $\rightarrow \mathrm{II}^{\prime} \rightarrow \mathrm{I}^{\prime}$ ) to generate the final product, which is identical to the starting reactants in a degenerate exchange process. (B) Reaction coordinate diagram showing the change in activation energies corresponding to structural changes, i.e., introduction of a proximal bulky group (left) or proximal basic group (right) in comparison to phenyl boronic ester (middle). For simplicity, the reaction coordinate diagram shows only half of the reaction up to the bis-diol trigonal intermediate, since the reverse reactions to form the boronic ester product are identical, based on microscopic reversibility.

covalent reaction. ${ }^{17-21}$ Therefore, PIC operates by the same principles as the well-established photoswitchable external catalysis, but enables catalysis by mildly acidic or basic groups that would not operate under intermolecular conditions.

Here we report a PIC that is capable of tuning the exchange rate between boronic ester and free diol over at least 4 orders of magnitude. The design of our photoswitch consists of two key components: first, the internal catalytic nitrogen in 8-quinoline boronic ester (8-QBE, Figure 1A), which assists in the rapid exchange between boronic ester and free diol; second, an acylhydrazone photoswitch that bears an acidic $\mathrm{N}-\mathrm{H}$, which forms an intramolecular $\mathrm{H}$-bond when positioned ortho to a basic heterocycle (Figure 1B). Unification of these two moieties yields the PIC system in Figure 1C. In its thermodynamically favored isomer, (E)-PIC, the photoswitch is in the "ON" state, in which internal catalysis from the proximal quinoline mediates rapid boronic ester exchange. Photoisomerization of the acyl-hydrazone to the "OFF" state, $(\boldsymbol{Z})$ PIC, results in the formation of an intramolecular H-bond between the quinoline nitrogen lone pair and the acyl-hydrazone $\mathrm{N}-\mathrm{H}$, deactivating internal catalysis, resulting in a drastically slower exchange.

Boronic ester transesterification represents an ideal chemistry to demonstrate PIC due to its wide dynamic range. In 1984, Wulff reported that the rate of exchange of boronic ester with diols spans a remarkable 14 orders of magnitude depending on the structure of the boronic ester. ${ }^{22}$ The exchange reaction proceeds via three fundamental steps: (I) addition, (II) proton transfer, and (III) elimination (Figure 2A). A small change in structure can alter the identity of the rate-limiting step, resulting in a dramatic change in rates (Figure $2 \mathrm{~B}$ ). At $20{ }^{\circ} \mathrm{C}$ in acetone, phenylboronic acid 1,2propanediol ester displays a modest exchange rate of $10^{-2} \mathrm{~s}^{-1}$, and proton transfer was determined to be rate limiting (II $>$ III $>$ I) based on a large kinetic isotope effect $\left(k_{\mathrm{H}} / k_{\mathrm{D}}=4-5\right)$. An increase in steric hindrance slows the exchange rate by as much as 4 orders of magnitude by increasing the barrier for addition (I $>$ II $>$ III). On the other hand, installing a proximal basic group significantly decreases the barrier for proton transfer through internal catalysis, making elimination rate limiting (III $>$ II $>$ I, no $\mathrm{KIE}$ ) and increasing the exchange rate by up to $10^{9}$-fold relative to phenylboronic ester.

The dramatic rate enhancement observed in 8-QBE can be attributed to the proximity of the quinoline nitrogen lone pair in relation to the boronic ester ${ }^{23,24}$ and the rigid aromatic structure. ${ }^{25}$ This structure allows the quinoline lone pair to form a 6-membered ring in the proton transfer transition state. The commonly used 2aminomethyl "Wulff-type" phenylboronic ester undergoes exchange an order of magnitude slower than $\mathbf{8 - Q B E}$, despite the more basic 


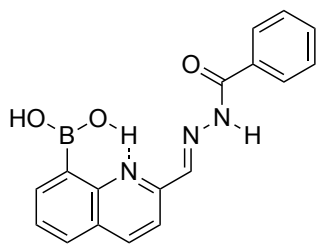

(E)-PIC acid

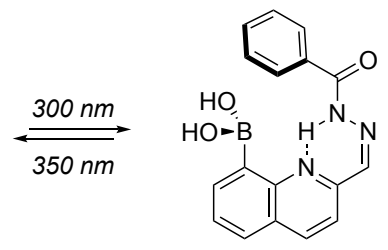

(Z)-PIC acid

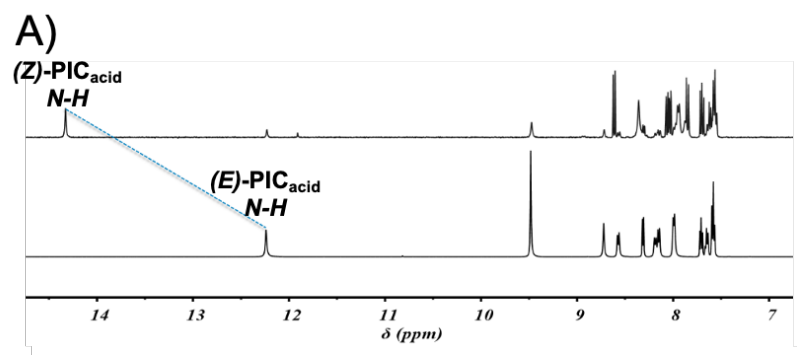

B)

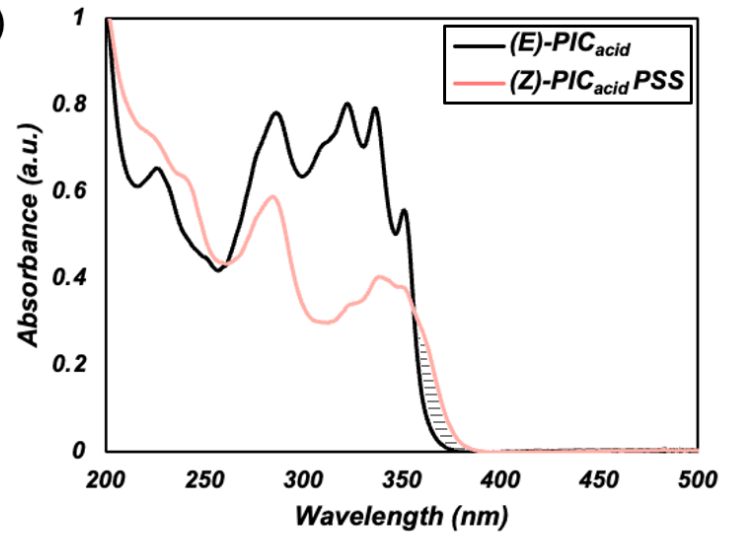

C)

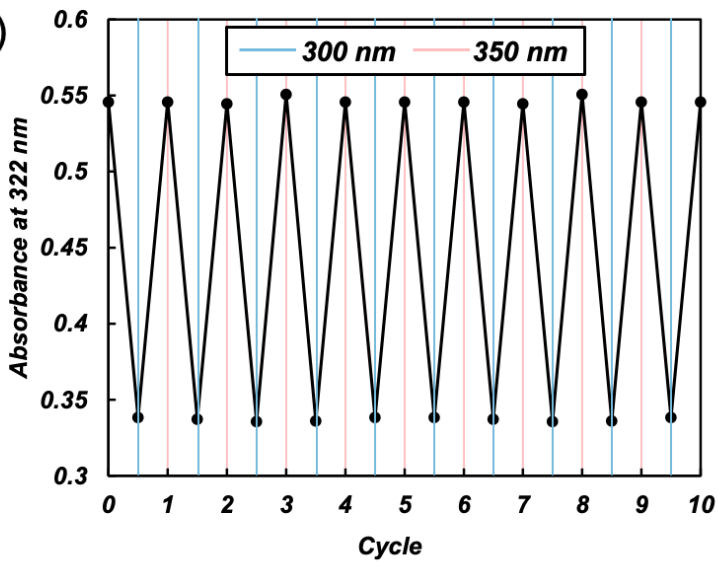

Figure 3. A) ${ }^{1} \mathrm{H}$ NMR of the aromatic region of $(\boldsymbol{E})$ - and (Z)-PIC acid shows the $\mathrm{N}-\mathrm{H}$ peak shift downfield after isomerization from $E$ to $Z$. B) UV-Vis absorbance of (E)-PICacid (black line) and the photostationary state of $(\boldsymbol{Z})$-PIC acid photostationary state (red line) in acetonitrile at $\left.1.56 \times 10^{-5} \mathrm{M}\right)$. C) Corresponding absorbance of

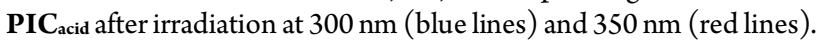

nitrogen, highlighting the importance of entropy in neighboring group participation.
We imagined that the rate-limiting step of boronic ester exchange could be remotely tuned with an appropriate photoswitch, thus dramatically altering the rate of exchange. Our strategy was to deactivate internal catalysis in $\mathbf{8 - Q B E}$ by engaging the quinoline lone pair in an intramolecular $\mathrm{H}$-bond. This intramolecular $\mathrm{H}$-bond, in turn, could be controlled by light using a suitable photoswitch. Our attention was drawn to a relatively novel class of acyl-hydrazone photoswitches that bear an acidic amide $\mathrm{N}-\mathrm{H}$ bond. ${ }^{26-32}$ Hydrazone photoswitches derived from 2-pyridinecarboxyaldehyde or 2quinolinecarboxaldehyde are thermally stable in the $Z$ isomer thanks to the formation of a six-membered intramolecular H-bond. Leigh has exploited the 2-pyridyl hydrazone photoswitch to control the activity of a linked squaramide organocatalyst through the position of an inhibiting group, achieving ca. 10-fold differences in conjugate addition rates, albeit requiring acid for the $Z \rightarrow E$ isomerization. ${ }^{33} \mathrm{We}$ envisioned that the intramolecular $\mathrm{H}$-bond, in addition to stabilizing the $Z$-isomer, could also turn "OFF" internal catalysis in 8-QBE. This design yielded PIC (Figure 1), wherein exchange is accelerated by internal catalysis when the hydrazone adopts the $E$ configuration and dramatically slowed in the $Z$ isomer.

Photoswitch properties. We synthesized (E)-PIC acid $_{\text {in }}$ its boronic acid form in 6 steps from 2-bromoaniline and crotonaldehyde (see SI for details). First, we investigated its photoisomerization by monitoring the conversion from $E$ to $Z$ by ${ }^{1} \mathrm{H}$ NMR. Initially, a sharp singlet appears at $12.2 \mathrm{ppm}$ in the E-isomer, corresponding to the acyl hydrazone $\mathrm{N}-\mathrm{H}$ bond. After irradiation at $300 \mathrm{~nm}$, the peak shifts downfield to $14.5 \mathrm{ppm}$, indicating the formation of a strong intramolecular $\mathrm{H}$-bond (Figure $3 \mathrm{~A}$ ). The $\mathrm{Z}$ isomer is thermally stabilized due to the intramolecular $\mathrm{H}$-bond and can be stored for weeks with no observable back isomerization, even when subjected to elevated temperatures $\left(70^{\circ} \mathrm{C}\right)$. Irradiation at 300 $\mathrm{nm}$ promotes efficient conversion from $(\boldsymbol{E})-\mathbf{P I C}_{\text {acid }} \rightarrow(\boldsymbol{Z})$-PIC $\mathbf{P I i d}_{\text {aci }}$ reaching a photostationary state (PSS) of $92 \%$. Although the $Z$ isomer is thermally stable, when irradiated with $350 \mathrm{~nm}$ light, the $Z \rightarrow E$ isomerization proceeds with a PSS of $67 \%$.

The fatigue resistance of other hydrazone photoswitches is generally quite high (up to 300 cycles). ${ }^{30,32}$ UV-Vis absorption of

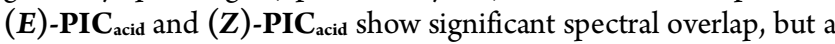
10 -nm bathochromic shift in the absorption onset of the $Z$ isomer provides enough spectral separation to selectively irradiate both isomers (Figure 3B). Using UV-Vis, we monitored $E \rightarrow Z$ isomerization over time and found that the PSS is reached in less than 1 minute in acetonitrile at $1.56 \times 10^{-5} \mathrm{M}$ (SI, Figure S1). $Z \rightarrow E$ isomerization also reaches (E)-PIC acid PSS in less than 2 minutes, enabling rapid switching between both isomers. Monitoring the UVVis absorption of $\mathbf{P I C}_{\text {acid }}$ at $322 \mathrm{~nm}$ during alternating irradiation at 300 and $350 \mathrm{~nm}$, we observe no loss in efficiency after 10 cycles under ambient conditions (Figure 3C). Therefore, the presence of a boronic acid does not affect the robustness of the hydrazone photoswitch.

Gratifyingly, after condensation with neopentyl glycol in the presence of excess diol (10 equiv), when boronic ester (E)-PIC $\mathbf{C}_{\text {ester }}$ is irradiated with $300 \mathrm{~nm}$ light, quantitative conversion to $(Z)$ PIC $_{\text {ester }}$ is observed (Figure S2). Likewise, in the presence of excess diol, quantitative conversion from $(\boldsymbol{Z})-\mathbf{P I C}_{\text {ester }}$ to $(\boldsymbol{E})-\mathbf{P I C}_{\text {ester }}$ is observed. This observation was rationalized by UV-Vis spectroscopy: when $(\boldsymbol{Z})$-PIC $\mathbf{P a c i d}_{\text {is }}$ converted to $(\boldsymbol{Z})$-PIC $\mathbf{P}_{\text {ester, }}$ we observe a $10 \mathrm{~nm}$ bathochromic shift in the absorption onset. In 

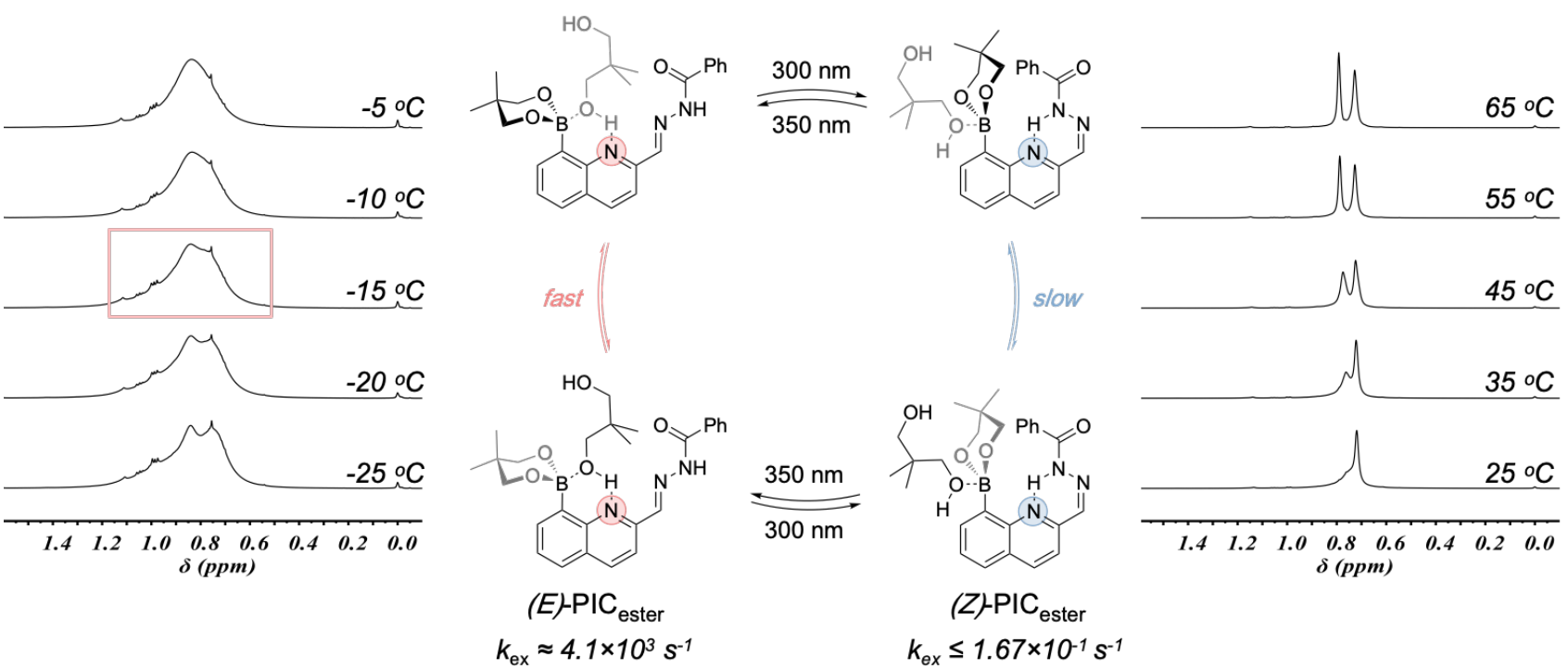

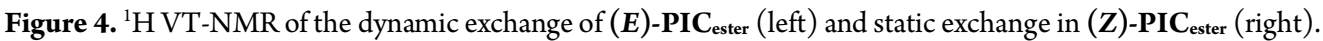

contrast, when comparing (E)-PIC acid to $(\boldsymbol{E})$-PIC $\mathbf{P}_{\text {ester, }}$ no significant spectral changes are observed by UV-Vis (Figure S3). Consequently, in the presence of excess diol, the boronic esters possess slightly improved spectral separation between the $E$ and $Z$ isomers compared to the boronic acids, enabling near-quantitative bidirectional switching.

Photoswitching exchange rates. We tested the effect of photoisomerization on the degenerate exchange between neopentyl glycol and the corresponding boronic ester (E)-PIC ester $(1: 1,100$ $\mathrm{mM})$. Toluene was used as the solvent, and a small amount of acetone was added to fully dissolve the diol (9:1 toluene-acetone). At $25{ }^{\circ} \mathrm{C},{ }^{1} \mathrm{H}$ NMR of $(\boldsymbol{E})$-PIC ${ }_{\text {ester }}$ shows a broad peak around 1.0 ppm, signifying that the dynamic exchange between diol and ester is occurring faster than the NMR timescale at this temperature. After irradiation with $300 \mathrm{~nm}$ light to achieve quantitative conversion to

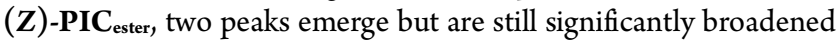
at room temperature. Upon heating the sample (up to $75^{\circ} \mathrm{C}$ ), two peaks emerge corresponding to the diol and ester, indicating that exchange has been considerably slowed, even at elevated temperatures.

The rate of exchange in (E)-PIC ester could be determined through coalescence between $-\mathrm{CH}_{3}$ resonances in bound and unbound neopentyl glycol by variable-temperature ${ }^{1} \mathrm{H}$ NMR (VT-NMR). Upon cooling the mixture, we observe the coalescence temperature of the bimolecular degenerate exchange to be $-15^{\circ} \mathrm{C}$ (Figure 5). The rate of exchange for $(\boldsymbol{E})$-PIC $\mathbf{C}_{\text {ester }}$ was thus determined to be 4.1 $\mathrm{x} 10^{3} \mathrm{~s}^{-1}$ at $25^{\circ} \mathrm{C}$ with an activation energy of $12.4 \mathrm{kcal} / \mathrm{mol}$ (see SI for details). A second coalescence temperature is observed at lower temperatures due to a unimolecular fluxional ring flip of the neopentylglycol boronate (Figure S4). ${ }^{34}$

Fluxionality due to the ring flip is also observed in (Z)-PIC $\mathbf{C}_{\text {ester, }}$ with coalescence occurring around $25^{\circ} \mathrm{C}$ (Figure S5). Upon further heating the sample, two peaks emerge as the ring flip becomes faster than the NMR timescale. The bimolecular exchange in (Z)-PIC $\mathbf{C}_{\text {ester }}$ however, was sufficiently slow that a coalescence temperature could not be observed even at elevated temperatures (Figure 5). From this, we can conclude that the exchange is occurring at least as slow as $1.67 \times 10^{-1} \mathrm{~s}^{-1}$ (calculated from $\mathrm{T}_{\mathrm{c}} \geq 75^{\circ} \mathrm{C}$ ). The exchange was not resolvable by exchange spectroscopy (EXSY) NMR (Figure S8). ${ }^{35}$

Thus, we have established an upper bound for the exchange rate

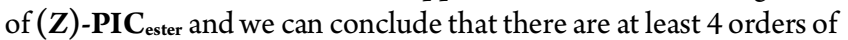

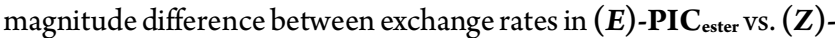
PIC $_{\text {ester. }}$ The fast bimolecular exchange in (E)-PIC ester suggests that internal catalysis reduces the activation energy for proton transfer. On the other hand, the slower bimolecular exchange in (Z)-PIC $\mathbf{C}_{\text {ester }}$ can be ascribed to the deactivation of internal catalysis through the formation of an intramolecular H-bond, increasing the barrier for proton transfer.

We have demonstrated that photoisomerization of a hydrazone can switch "OFF" internal catalysis of boronic ester exchange. The resulting $10^{4}$ difference in exchange rates is enabled by the formation of an intramolecular $\mathrm{H}$-bond. To show that chemical stimuli cannot regulate internal catalysis to the same extent, we synthesized control (2-methylquinolin-8-yl)boronic acid (Me-QBA, SI). VT-NMR revealed that the corresponding neopentyl glycol boronate, $\mathbf{M e}$ QBE undergoes exchange roughly 10 times faster than to $(\boldsymbol{E})$ PIC $_{\text {ester }}\left(4.9 \times 10^{4} \mathrm{~s}^{-1}\right.$ at $25{ }^{\circ} \mathrm{C}$, Figure S6). We hypothesize that exchange in (E)-PIC $\mathbf{C}_{\text {ester }}$ is slower than that of Me-QBE due to the presence of the electron-withdrawing hydrazone, which reduces the basicity of the quinoline, and contributes some degree of steric hindrance. When Me-QBE is exposed to 1.0 equivalent of trifluoroacetic acid (TFA), an acid capable of fully protonating the quinoline, only a moderate decrease in exchange rates was observed $\left(3.0 \times 10^{4} \mathrm{~s}^{-1}\right.$, Figure S7). This observation is consistent with the fact that diol addition to boronic acids can be both acid and base catalyzed, ${ }^{36,37}$ so external proton sources cannot deactivate internal catalysis. These experiments further highlight the importance the intramolecular $\mathrm{H}$-bond in our design to deactivate internal catalysis. Additionally, Letsinger has shown that the presence of exogeneous quinoline does not increase the transesterification rate for phenylboronic ester, indicating that internal catalysis is crucial for accelerating exchange. ${ }^{23}$ 
Conclusion. We have demonstrated the use of a thermally stable, bidirectional hydrazone photoswitch to control the rate of exchange between a boronate ester and the corresponding diol by at least 4 orders of magnitude. The dramatic change in rates afforded by reversible deactivation of internal catalysis lays the foundation for photocontrolling kinetics in different dynamic covalent reactions. The ability to remotely and reversibly control a dynamic covalent exchange rate can be translated to turn on and off assembly and reconfiguration in smart materials. The application of these photoswitchable dynamic bonds to tune viscoelasticity in polymer networks is ongoing in our laboratory.

\section{ASSOCIATED CONTENT}

Supporting Information. Synthetic procedures; NMR data; photochemical characterization. This material is available free of charge via the Internet at http://pubs.acs.org

\section{AUTHOR INFORMATION}

\section{Corresponding Author}

*jkalow@northwestern.edu

\section{ACKNOWLEDGMENT}

This material is based upon work supported by the National Science Foundation under CAREER CHE-1847948 and a 3M Non-Tenured Faculty Award (J.A.K.). This work made use of NMR and MS instrumentation at the Integrated Molecular Structure Education and Research Center (IMSERC) at Northwestern, which has received support from the NSF (NSF CHE-9871268); Soft and Hybrid Nanotechnology Experimental (SHyNE) Resource (NSF ECCS1542205); the State of Illinois and International Institute for Nanotechnology.

\section{REFERENCES}

1. Winne, J. M.; Leibler, L.; Du Prez, F. E. Dynamic covalent chemistry in polymer networks: a mechanistic perspective. Polym. Chem. 2019, 10, 6091-6108.

2. Wojtecki, R. J.; Meador, M. A.; Rowan, S. J. Using the dynamic bond to access macroscopically responsive structurally dynamic polymers. Nat. Mater. $2011,10,14-27$.

3. Boehnke, N.; Cam, C.; Bat, E.; Segura, T.; Maynard, H. D. Imine Hydrogels with Tunable Degradability for Tissue Engineering. Biomacromolecules 2015, 16, 2101-2108.

4. Lohse, M. S.; Bein, T. Covalent Organic Frameworks: Structures, Synthesis, and Applications. Adv. Funct. Mater. 2018, 28, 1705553.

5. Liu, Y.; Lehn, J. M.; Hirsch, A. K. H. Molecular Biodynamers: Dynamic Covalent Analogues of Biopolymers. Acc. Chem. Res. 2017, 50, 376-386.

6. Rottger, M.; Domenech, T.; van der Weegen, R.; Breuillac, A.; Nicolay, R.; Leibler, L. High-performance vitrimers from commodity thermoplastics through dioxaborolane metathesis. Science 2017, 356, 62-65.

7. Gu, R. R.; Flidrova, K.; Lehn, J. M. Dynamic Covalent Metathesis in the $\mathrm{C}=\mathrm{C} / \mathrm{C}=\mathrm{N}$ Exchange between Knoevenagel Compounds and Imines. J. Am. Chem. Soc. 2018, 140, 5560-5568.

8. Kathan, M.; Hecht, S. Photoswitchable molecules as key ingredients to drive systems away from the global thermodynamic minimum. Chem. Soc. Rev., 2017, 46, 5536-5550.
9. Accardo, J. V.; McClure, E. R.; Mosquera, M. A.; Kalow, J.A. Using Visible Light to Tune Boronic Acid-Ester Equilibria. J. Am. Chem. Soc. 2020, 142, 19969-19979.

10. Lemieux, V.; Gauthier, S.; Branda, N. R. Selective and Sequential Photorelease Using Molecular Switches. Angew. Chem. 2006 118, 6974-6978.

11. Göstl, R; Hecht, S. Controlling covalent connection and disconnection with light. Angew. Chem. Inter. Ed. 2014, 53, 87848787.

12. Kathan, M.; Eisenreich, F.; Jurissek, C.; Dallmann, A.; Gurke, J.; Hecht, S. Light-driven molecular trap enables bidirectional manipulation of dynamic covalent systems. Nat. Chem. 2018, 10, 1031-1036.

13. Wilson, D.; Branda, N. R. Turning "on" and "off" a pyridoxal 5'phosphate mimic using light. Angew. Chem. Inter. Ed. 2012, 51, 5431-5434.

14. Kathan, M.; Kovaříček, P.; Jurissek, C.; Senf, A.; Dallmann, A.; Thünemann, A. F.; Hecht, S. Control of Imine Exchange Kinetics with Photoswitches to Modulate Self-Healing in Polysiloxane Networks by Light Illumination. Angew. Chem. Int. Ed. 2016, 55, 13882-13886.

15. Neilson, B. M.; Bielawski, C. W. lluminating photoswitchable catalysis. ACS Cat. 2013, 3, 1874-1885.

16. Dorel, R.; Feringa, B. L. Photoswitchable catalysis based on the isomerisation of double bonds. Chem. Comm. 2019, 55, 64776486.

17. Cromwell, O. R.; Chung, J.; Guan, Z. Malleable and Self-Healing Covalent Polymer Networks through Tunable Dynamic Boronic Ester Bonds. J. Am. Chem. Soc. 2015, 137, 6492-6495.

18. Nishimura, Y.; Chung, J.; Muradyan, H.; \& Guan, Z. Silyl Ether as a Robust and Thermally Stable Dynamic Covalent Motif for Malleable Polymer Design. J. Am. Chem. Soc. 2017, 139, 1488114884.

19. Delahaye, M.; Winne, J. M.; Du Prez, F. E. Internal Catalysis in Covalent Adaptable Networks: Phthalate Monoester Transesterification As a Versatile Dynamic Cross-Linking Chemistry. J. Am. Chem. Soc. 2019 141, 15277-15287.

20. Van Lijsebetten, F.; Holloway, J. O.; Winne, J. M.; Du Prez, F. E. Internal catalysis for dynamic covalent chemistry applications and polymer science. Chem. Soc. Rev. 2020, 49, 8425-8438.

21. Cuminet, F., Caillol, S.; Dantras, E.; Leclerc, E.; Ladmiral, V. Neighboring Group Participation and Internal Catalysis Effects on Exchangeable Covalent Bonds: Application to the Thriving Field of Vitrimer Chemistry. Macromolecules 2021, 54, 3927-3961.

22. Wulff, G.; Lauer, M.; and Bohnke, H. Rapid Proton Transfer as Cause of an Unusually Large Neighboring Group Effect. Angew. Chem. Int. Ed. 1984, 23, 741-742.

23. Letsinger, R. L.; Dandegaonke, S. H. Organoboron Compounds. IX. 8-Quinolineboronic Acid, its Preparation and Influence on Reactions of Chlorohydrins, J. Am. Chem. Soc. 1958, 81, 498-501.

24. Morrison J. D.; Letsinger, R. Organoboron Compounds. XVIII. Bifunctional Binding of Water by the cis-1,2-Cyclopentanediol Ester of 8-Quinolineboronic Acid L. J. Org. Chem. 1964, 29, 11, 3405-3407

25. Goldman, M.; Wehry, E. L. Environmental effects upon the photoluminescence of 8-quinolineboronic acid Anal. Chem. 1970, $42,11,1186-1188$

26. Su, X.; Aprahamian, I. Hydrazone-based switches, metalloassemblies and sensors. Chem. Soc. Rev. 2014, 43, 1963-1981.

27. Chaur, M. N.; Collado, D.; \& Lehn, J. M. Configurational and constitutional information storage: Multiple dynamics in systems 
based on pyridyl and acyl hydrazones. Chem. A Eur. J. 2011, 17, 248-258.

28. Vantomme, G.; Hafezi, N.; Lehn, J. M. A light-induced reversible phase separation and its coupling to a dynamic library of imines. Chem. Sci. 2014, 5, 1475-1483.

29. Su, X.; Aprahamian, I. Hydrazone-based switches, metalloassemblies and sensors. Chem. Soc. Rev. 2014, 43, 1963-1981.

30. Van Dijken, D. J.; Kovaříček, P.; Ihrig, S. P.; Hecht, S. Acylhydrazones as Widely Tunable Photoswitches. J. Am. Chem. Soc. 2015, 137, 14982-14991.

31. Romero, E. L.; D’Vries, R. F.; Zuluaga, F.; Chaur, M. N. Multiple dynamics of hydrazone based compounds. J. Braz. Chem. Soc. 2015, 26, 1265-1273.

32. Qian, H.; Pramanik, S.; Aprahamian, I. Photochromic Hydrazone Switches with Extremely Long Thermal Half-Lives. J. Am. Chem. Soc, 2017 139, 9140-9143.

33. De Bo, G.; Leigh, D. A.; McTernan, C. T.; Wang, S. $\underline{A}$ complementary pair of enantioselective switchable organocatalysts Chem. Sci. 2017, 8, 7077-7081.

34. Eichhorn, A. Copper(I) catalyzed borylation and cross-coupling reactions; (Figure 45, page 99) $\mathrm{PhD}$ Thesis, Julius Maximilian University of Würzburg: Würzburg, Germany, 2018.

35. Kleckner, I. R; \& Foster, M. P. (2011). An introduction to NMRbased approaches for measuring protein dynamics. Biochimica et Biophysica Acta 2011, 1814, 942-968.

36. Collins, B. E.; Metola, P.; Anslyn, E. V. On the rate of boronate ester formation in ortho-aminomethyl-functionalised phenyl boronic acids. Supramol. Chem. 2013, 25, 79-86.

37. Sun, X.; Chapin, B. M.; Metola, P.; Collins, B.; Wang, B.; James, T. D.; Anslyn, E. V. The mechanisms of boronate ester formation and fluorescent turn-on in ortho-aminomethylphenylboronic acids. Nat. Chem. 2019, 11, 768-778. 